Vol. XX (XXXX)

No. X

\title{
SIMPLE CUBIC RANDOM-SITE PERCOLATION THRESHOLDS FOR COMPLEX NEIGHBOURHOODS
}

\author{
Łukasz Kurzawski and Krzysztof Malarz* \\ AGH University of Science and Technology, \\ Faculty of Physics and Applied Computer Science, \\ al. Mickiewicza 30, 30-059 Krakow, Poland. \\ e-mail: malarz@agh.edu.pl
}

(Received December 8, 2011)

\begin{abstract}
In this communication with computer simulation we evaluate simple cubic randomsite percolation thresholds for neighbourhoods including the nearest neighbours $(\mathrm{NN})$, the next-nearest neighbours $(2 \mathrm{NN})$ and the next-next-nearest neighbours $(3 \mathrm{NN})$. Our estimations base on finite-size scaling analysis of the percolation probability vs. site occupation probability plots. The Hoshen-Kopelman algorithm has been applied for cluster labelling. The calculated thresholds are 0.1372(1), 0.1420(1), 0.0976(1), 0.1991(1), 0.1036(1), 0.2455(1) for $(\mathrm{NN}+2 \mathrm{NN}),(\mathrm{NN}+3 \mathrm{NN}),(\mathrm{NN}+$ $2 \mathrm{NN}+3 \mathrm{NN}), 2 \mathrm{NN},(2 \mathrm{NN}+3 \mathrm{NN}), 3 \mathrm{NN}$ neighbourhoods, respectively. In contrast to the results obtained for a square lattice the calculated percolation thresholds decrease monotonically with the site coordination number $z$, at least for our inspected neighbourhoods.
\end{abstract}

Keywords: site percolation; percolation thresholds; computer simulations

\section{Introduction}

Percolation [1]2] is a mathematical description of a geometrical phase transition. This allows for purely theoretical studies of all phenomena occurring near the critical point with computer experiments solely or, sometimes, even analytically [3]. The mixture of occupied and empty sites of given lattice may exhibit some features of real physical systems. Among typical applications of the percolation theory one may find material science [4], immunology [5] or forest fires problems [6] and studies of liquids moving in porous media [7, etc. [8, 9].

Generally speaking, the percolation theory deals with statistical properties of the clusters of occupied nodes (site percolation) or occupied edges (bond percolation) for a given graph, network or regular lattice. In the site percolation problem, the cluster is defined as a group of the occupied lattice vertexes which are direct or indirect neighbours. When each site is occupied with some probability $p$ there is a critical probability

* http://home.agh.edu.pl/malarz/ 
of sites occupation $p_{c}$ above which a cluster spanning through the whole system appears for the first time [8, 9. This special probability is called percolation threshold $p_{c}$ and it separates two phases (in the language of material science a conductor and an isolator). The value of percolation threshold $p_{c}$ depends on kind of percolation (site/bond), lattice/graph/network topology and assumed sites neighbourhoods. In the simplest case only the nearest neighbours constitute the neighbourhoods (von Neumann's neighbourhood) or the nearest neighbours and next-nearest neighbours are considered (Moore's neighbourhood).

In the vicinity of the phase transition the quantity $A$ describing the system follows a scaling relation

$$
A \propto L^{\alpha} \cdot f\left(x \cdot L^{\beta}\right)
$$

where $L$ describes the linear size of the system, $f(\cdot)$ is a scaling function and $x$ is dimensionless scaling parameter 10. For physical systems $x$ usually plays the role of reduced temperature $\left(T-T_{C}\right) / T_{C}$, where $T_{C}$ stands for critical temperature. The $\alpha$ and $\beta$ parameters are universal exponents which - in the first approximation - do not depend on system details (kind of order/disorder phenomenon, lattice shape, site or bond percolation, etc.) but only on the system dimensionality [8,10. However, for $\alpha$ and $\beta$ calculations the precise value of $T_{C}$ is required. For the geometrical model of the phase transition percolation threshold $p_{c}$ plays the role of critical temperature.

In this communication we evaluate with computer simulations [11] the random-site simple cubic percolation thresholds for neighbourhoods including the nearest-neighbours $(\mathrm{NN})$, the second-nearest neighbours $(2 \mathrm{NN})$ and the third-nearest neighbours $(3 \mathrm{NN})$. Our estimations base on finite size scaling analysis [8, 10, of the percolation probability vs. site occupation probability plots. The Hoshen-Kopelman algorithm [12] has been applied for cluster labelling. The calculated thresholds $p_{c}$ are 0.1372(1), 0.1420(1), 0.0976(1), $0.1991(1), 0.1036(1), 0.2455(1)$ for $(\mathrm{NN}+2 \mathrm{NN}),(\mathrm{NN}+3 \mathrm{NN}),(\mathrm{NN}+2 \mathrm{NN}+3 \mathrm{NN}), 2 \mathrm{NN}$, $(2 \mathrm{NN}+3 \mathrm{NN}), 3 \mathrm{NN}$ neighbourhoods, respectively. In contrast to the results obtained for a square lattice 13 15] the calculated percolation thresholds decrease monotonically with the site coordination number $z$, at least for our inspected neighbourhoods.

$(\mathrm{NN})$

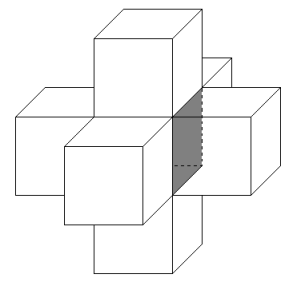

$(2 \mathrm{NN})$

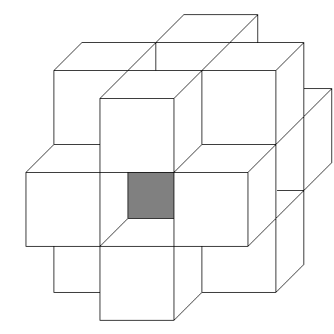

$(3 \mathrm{NN})$

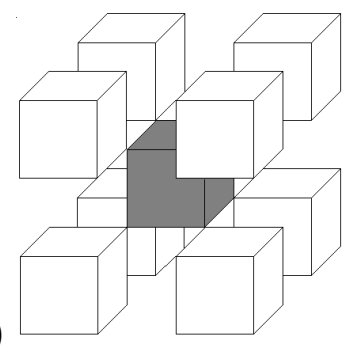

Fig. 1: Basic neighbourhoods for simple cubic lattice. The NN neighbourhood is often referred to as von Neumann's neighbourhood while combination of $(\mathrm{NN}+2 \mathrm{NN})$ is called Moore's neighbourhood. We propose to name $(\mathrm{NN}+2 \mathrm{NN}+3 \mathrm{NN})$ neighbourhood in simple cubic lattice the Rubik's neighbourhood. 


\section{Calculations}

We use Hoshen-Kopelman algorithm 12 for occupied sites labelling. In the HoshenKopelman scheme each site has one label: all sites in a given cluster have the same labels and different clusters have assigned different labels.

Examples of percolation probability $P$ vs. sites occupation probability $p$ for various neighbourhoods and for various lattice linear sizes $L$ are presented in Fig. 2. We use finite-size scaling analysis to determine $p_{c}$ numerically. As for finite systems the phase transition is never sharp we observe it when for increasing lattice sizes $L$ the $P(p)$ curves become more and more steep and intersect at a common point corresponding also to the case of $L \rightarrow \infty$ [16. The common cross-point predicts percolation threshold $p_{c}$. Such strategy was successfully applied for many system description where phase transition may be observed including percolation [17, Ising model [18, majority-vote models [19] or opinion dynamics 20 .

Basing on $P(p)$ dependence for various $L$ we look for an interval of the length $\Delta p=$ $2 \cdot 10^{-4}$ where curves for $L=63$ and 100 cross each other. The results have been averaged over $N=10^{5}$ lattice realisations. For example for $2 \mathrm{NN}$ case this interval is $(0.1990,0.1992)$ and the centre of this bracket plays the role of percolation threshold. Basing on a uncertainty type $\mathrm{B}$ evaluation procedure [21] we estimate the uncertainty $u\left(p_{c}\right)=\Delta p / \sqrt{3} \approx 10^{-4}$.

\section{Results}

The $P(p)$ dependencies for $L=22,63$ and 100 are presented in Fig. 2, The evaluated percolation thresholds $p_{c}$ for various neighbourhoods are collected in Tab. 1. To check the accuracy of our estimations we evaluated the percolation threshold for compact neighbourhoods as well. The obtained values $p_{c}(\mathrm{NN})=0.3116(1), p_{c}(\mathrm{NN}+2 \mathrm{NN})=0.1372(1)$ and $p_{c}(\mathrm{NN}+2 \mathrm{NN}+3 \mathrm{NN})=0.0976(1)$ agree nicely with the results of extensive numerical simulations 22] and the earlier estimations 23,24.

Table 1: Simple cubic lattice random-site percolation thresholds $p_{c}$ for various neighbourhoods constructed with basic neighbourhoods NN, 2NN and 3NN.

\begin{tabular}{lrll}
\hline \hline neighbourhood & $z$ & $p_{c}$ & earlier estimations \\
\hline NN & 6 & $0.3116(1)$ & $0.31160 \cdots[22]$ \\
$2 \mathrm{NN}$ & 12 & $0.1991(1)$ & \\
$3 \mathrm{NN}$ & 8 & $0.2455(1)$ & \\
$\mathrm{NN}+2 \mathrm{NN}$ & 18 & $0.1372(1)$ & $0.137[23,0.13735(5)[24]$ \\
$\mathrm{NN}+3 \mathrm{NN}$ & 14 & $0.1420(1)$ & \\
$2 \mathrm{NN}+3 \mathrm{NN}$ & 20 & $0.1036(1)$ & \\
$\mathrm{NN}+2 \mathrm{NN}+3 \mathrm{NN}$ & 26 & $0.0976(1)$ & $0.097[23,0.0976445(10)[24]$ \\
\hline \hline
\end{tabular}


$(2 \mathrm{NN})$

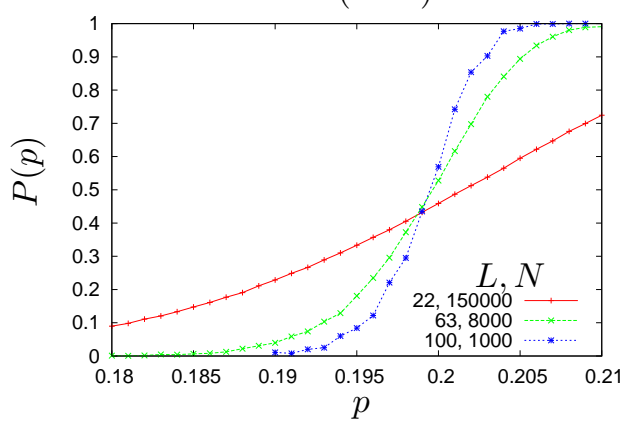

$(\mathrm{NN}+2 \mathrm{NN})$

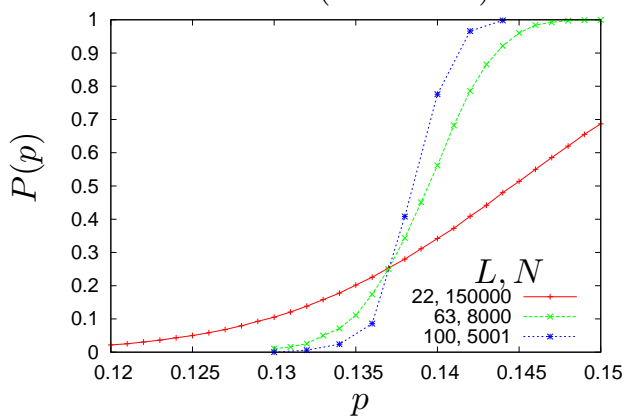

$(2 \mathrm{NN}+3 \mathrm{NN})$

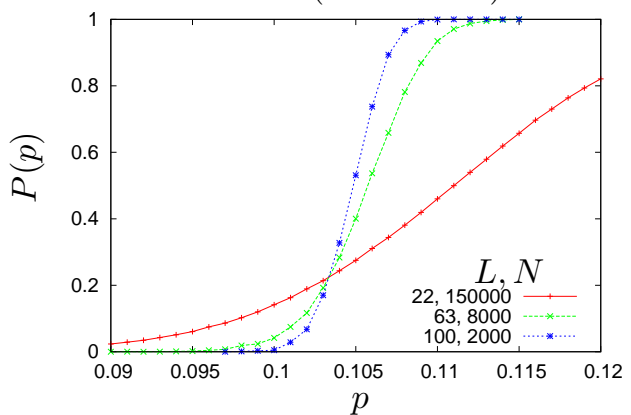

(3NN)

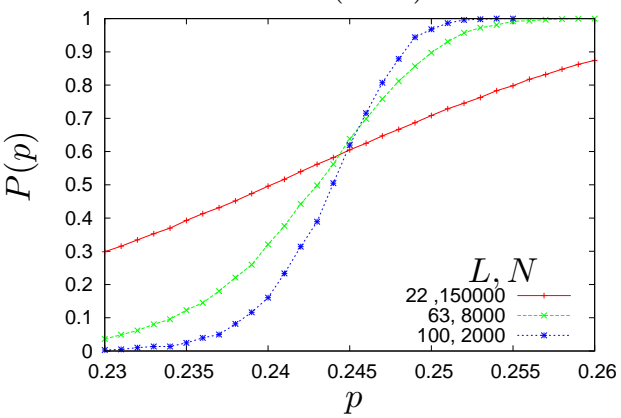

$(\mathrm{NN}+3 \mathrm{NN})$

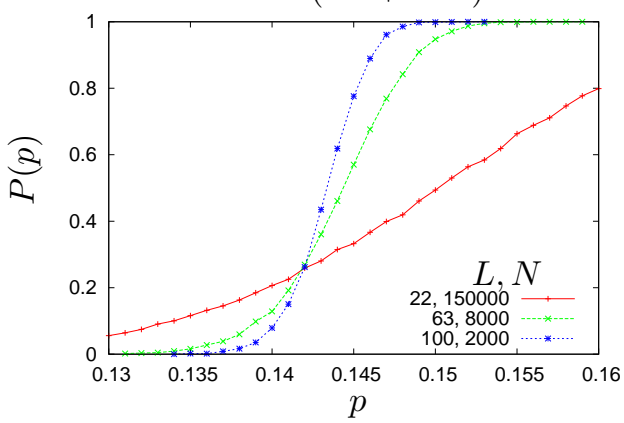

$(\mathrm{NN}+2 \mathrm{NN}+3 \mathrm{NN})$

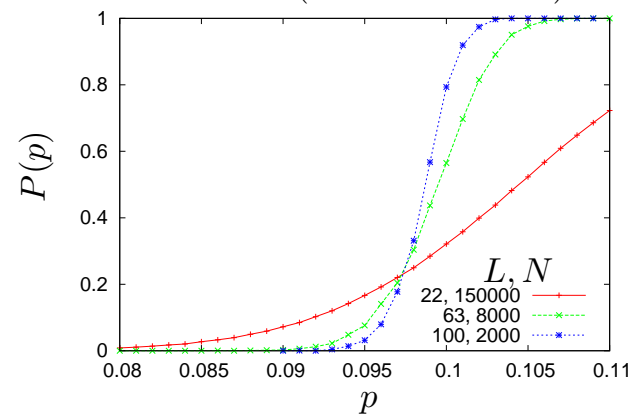

Fig. 2: Percolation probability $P$ vs. site occupation probability $p$ for various neighbourhoods in three dimensions for three lattice sizes $L$. The results presented here have been averaged over $N$ lattice realisations. 


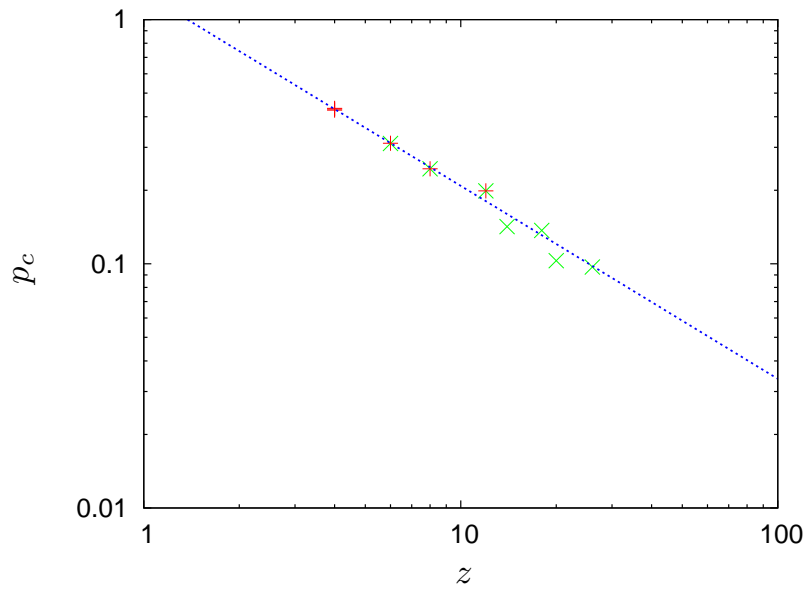

Fig. 3: Percolation thresholds $p_{c}$ vs. sites coordination number $z$ for our $(\times)$ inspected three-dimensional neighbourhoods and other three-dimensional lattices 25-31] estimations $(+)$. The straight lines are the least squares fits of $p_{c}(z) \propto z^{-\gamma}$ to the experimental data.

These $p_{c}$ values decrease with sites coordination number $z$ as shown in Fig. 3 The $p_{c}(z)$ dependencies may be fitted nicely by a straight line in a logarithmic plot, namely: $p_{c}(z) \propto z^{-\gamma}$ with $\gamma=0.790(26)$. The percolation thresholds for some three-dimensional lattices have been used for fitting $p_{c}(z)$ dependence including ice [25], diamond [26, 27], hpc [28, bcc [27, 29, 30, fcc [27,30, and $\mathrm{La}_{2-x} \mathrm{Sr}_{x} \mathrm{CuO}_{4}$,31] lattices. These additional percolation threshold values are indicated as pluses $(+)$ in Fig. 3

\section{Conclusions}

In this communication for the first time we evaluate the random-site percolation thresholds for the simple cubic lattice with $2 \mathrm{NN}, 3 \mathrm{NN}, \mathrm{NN}+3 \mathrm{NN}$ and $2 \mathrm{NN}+3 \mathrm{NN}$ neighbourhoods for which sites from the first, the second and the third coordination shells were included (see Tab. 1). The obtained thresholds $p_{c}$ decrease monotonically with sites coordination number $z$ according to the power law $p_{c} \propto z^{-\gamma}$, with exponent $\gamma=0.790(26)$.

In contrast to the results obtained for a square lattice [15] the calculated percolation thresholds decrease monotonically with the site coordination number $z$, at least for inspected neighbourhoods.

The obtained results may be helpful in studies of the universal formulae [32] for percolation thresholds $p_{c}$ dependence on sites coordination number $z$.

Finally, we propose to name $(\mathrm{NN}+2 \mathrm{NN}+3 \mathrm{NN})$ neighbourhood in simple cubic lattice the Rubik's neighbourhood as it is identical with the famous Rubik's cube [33] - a very popular logical puzzle in early 80 's. 


\section{Acknowledgements}

We are grateful to Dietrich Stauffer and an anonymous Referee for paying our attention to Ref. [23] and Ref. 24], respectively. Supported by the Polish Ministry of Science and Higher Education and its grants for scientific research. The numerical calculations were carried out in the Academic Computer Centre CYFRONET-AGH (grants No. MEiN/SGI3700/AGH/024/2006 and MNiSW/Zeus_lokalnie/AGH/068/2011).

\section{REFERENCES}

[1] S. R. Broadbent, J. M. Hammersley: Proc. Cambridge Phil. Soc. 53, 629-641 (1957);

J. M. Hammersley: Proc. Cambridge Phil. Soc. 53, 642-645 (1957);

H. L. Frisch, J. M. Hammersley, D. J. A. Welsh: Phys. Rev. 126, 949-951 (1962).

[2] M. F. Sykes, M. Glen: J. Phys. A 9, 87-95 (1976);

M. F. Sykes, D. S. Gaunt, M. Glen: ibid. 9, 97-103 (1976);

M. F. Sykes, D. S. Gaunt, M. Glen: ibid. 9, 715-724 (1976);

M. F. Sykes, D. S. Gaunt, M. Glen: ibid. 9, 725-730 (1976);

D. S. Gaunt, M. F. Sykes: ibid. 9, 1109-1116 (1976).

[3] J. C. Wierman, S. P. Naor, R. Cheng: Phys. Rev. E 72, 066116 (2005);

A. Rosowsky: Eur. Phys. J. B 15, 77-86 (2000).

[4] B. I. Halperin and D. J. Bergman: Physica B 405, 2908-2914 (2010);

P. R. Shearing, D. J. L. Brett, N. P. Brandon: International Materials Reviews 55, 347-363 (2010);

J. Silva, R. Simoes, S. Lanceros-Mendez, R. Vaia: EPL 93, 37005 (2011).

[5] S. U. Suzuki, A. Sasaki J. Theor. Biol. 276, 117-125 (2011);

J. Lindquist, Junling Ma, P. van den Driessche, et al: J. Math. Biol. 62, 143-164 (2011);

E. N. Naumova, J. Gorski, Yu. N. Naumov: Annales Zoologici Fennici 45, 369-384 (2008);

W. Floyd, L. Kay, M. Shapiro: Bull. Math. Biol. 70, 713-727 (2008).

[6] G. Camelo-Neto, S. Coutinho: J. Stat. Mech., P06018 (2011);

N. Guisoni, E. S. Loscar, E. V. Albano: Phys. Rev. E 83, 011125 (2011);

A. Simeoni, P. Salinesi, F. Morandini: Int. J. Wildland Fire 20, 625-632 (2011);

K. Malarz, S. Kaczanowska, K. Kułakowski: Int. J. Mod. Phys. C 13, 1017-1031 (2002).

[7] Y. Amiaz, S. Sorek, Y. Enzel, et al: Water Resources Research 47, W10513 (2011);

S. F. Bolandtaba, A. Skauge: Transport in Porous Media 89, 357-382 (2011);

V. V. Mourzenko, J.-F. Thovert, P. M. Adler: Phys. Rev. E 84, 036307 (2011).

[8] D. Stauffer, A. Aharony: Introduction to Percolation Theory, Taylor and Francis, London 1994;

[9] H. Kesten: Percolation Theory for Mathematicians, Brikhauser, Boston 1982;

M. Sahimi: Applications of Percolation Theory, Taylor and Francis, London 1994;

D. Stauffer: Physica A 242, 1-7 (1997).

[10] D. P. Landau, K. Binder: A Guide to Monte Carlo Simulation in Statistical Physics, Cambridge University Press, Cambridge 2005. 
[11] E. Kurzawski: M.Sc. Thesis, AGH University of Science and Technology, Kraków 2011.

[12] J. Hoshen, R. Kopelman: Phys. Rev. B 14, 3438-3445 (1976).

[13] K. Malarz, S. Galam: Phys. Rev. E 71, 016125 (2005).

[14] S. Galam, K. Malarz: Phys. Rev. E 72, 027103 (2005).

[15] M. Majewski, K. Malarz: Acta Phys. Pol. B 38, 2191-2199 (2007).

[16] V. Privman (Ed.): Finite size scaling and numerical simulation of statistical systems, World Scientific, Singapore 1990.

[17] M. E. J. Newman, R. M. Ziff: Phys. Rev. E 85, 016706 (2001).

[18] F. W. S. Lima, J. Mostowicz, K. Malarz: Commun. Comput. Phys. 10, 912-919 (2011).

[19] J. C. Santos, F. W. S. Lima, K. Malarz: Physica A 390, 359-364 (2011);

F. W. S. Lima, K. Malarz: Int. J. Mod. Phys. C 17, 1273-1283 (2006);

F. W. S. Lima, A. O. Sousa, M. A. Sumuorc: Physica A 387, 3503-3510 (2008).

[20] D. Stauffer, A. O. Sousa, S. Moss de Oliveira: Int. J. Mod. Phys. C 11, 1239-1245 (2000); A. A. Moreira, J. S. Andrade Jr., D. Stauffer: Int. J. Mod. Phys. C 12, 39-42 (2001); A. O. Sousa: Physica A 348, 701-710 (2005).

[21] ISO Technical Advisory Group, Working Group 3: Guide to the Expression of Uncertainty in Measurement, International Organisation for Standardization, Geneva 1993; L. J. Gleser: Statist. Sci. 13, 277-290 (1998).

[22] P. Grassberger: J. Phys. A 25, 5867-5888 (1992);

C. D. Lorenz, R. M. Ziff: J. Phys. A 31, 8147-8157 (1998);

N. Jan, D. Stauffer: Int. J. Mod. Phys. C 9, 341-347 (1998);

P. N. Ballesteros, L. A. Fernandez, V. Martín-Mayor, et al: J. Phys. A 32, 1-13 (1999);

Youjin Deng, H. W. J. Blöte: Phys. Rev. E 72, 016126 (2005);

M. Acharyya, D. Stauffer: Int. J. Mod. Phys. C 9, 643-647 (2009);

J. Škvor, I. Nezbeda: Phys. Rev. E 79, 041141 (2009).

[23] C. Domb, N. W. Dalton: Proc. Phys. Soc. 89, 859-871 (1966).

[24] R. M. Ziff, S. Torquato: priv. commun.

[25] V. A. Vyssotsky, H. L. Frisch, E. Sonnenblick, J. M. Hammersley: Phys. Rev. 124, 10211022 (1961).

[26] A. Silverman, J. Adler: Phys. Rev. B 42, 1369-1373 (1990).

[27] S. C. van der Marck: Int. J. Mod. Phys. C 9, 529-540 (1998).

[28] C. D. Lorenz, R. May, R. M. Ziff: J. Stat. Phys. 98, 961-970 (2000).

[29] R. M. Bradley, P. N. Strenski, J.-M. Debierre: Phys. Rev. B 44, 76-84 (1991);

D. S. Gaunt, M. F. Sykes: J. Phys. A 16, 783-799 (1983).

[30] C. D. Lorenz, R. M. Ziff: J. Phys. A 31, 8147-8157 (1998).

[31] J. Tahir-Kheli, W. A. Goddard III: Phys. Rev. B 76, 014514 (2007).

[32] M. Sahimi, B. D. Hughes, L. E. Scriven, T. Davis: J. Phys. A 16, L67-L71 (1983);

S. Galam, A. Mauger: J. Appl. Phys. 75, 5526-5528 (1994);

S. Galam, A. Mauger: Physica A 205, 502-510 (1994);

S. C. van der Marck: Phys. Rev. E 55, 1228-1229 (1997).

[33] http://www.rubiks.com/ 